\title{
SENSOR BASED APPLICATION FOR SMART VEHICLES
}

Arjun $\mathrm{K}^{1}$, Prithviraj ${ }^{2}$ and Ashwitha $\mathrm{A}^{3}$

\begin{abstract}
The problem of vehicle accident is part of an endless list of disasters that could occur anywhere anytime. Statistically, they are considered the second leading cause of death. To overcome such problems, many vehicle manufacturers and automobile device companies have attempted to develop speed control systems to maintain a vehicle safe distance. The proposed project aim is to design and development of app for car driver. In this project we are presenting an IoT based system which will help drivers to drive the car safely and efficiently. This system consists of tracking and locating the location of accident using GPS and communicates the co-ordinates via SMS using onboard GSM module, smoke detection using a gas sensor, obstacle detection in front and rear of car using ultrasonic sensor and simulation of physically unavailable sensors using logical functions.
\end{abstract}

Keywords - Smoke sensors, Fire sensors, GSM module

\section{INTRODUCTION}

The problem of vehicle accident is part of an endless list of disasters that could occur anywhere anytime. According to the Association for Safe International Road Travel, about 1.24 million die and 50 million are injured on the roads of the world every year. Statistically, they are considered the second leading cause of death. To overcome such problems[1], many vehicle manufacturers and automobile device companies have attempted to develop speed control systems to maintain a vehicle safe distance. In this direction the attempt is going on designing a safety driving app for automobiles using the new emerging IOT based technology that is used for designing a more efficient solution[2]. The proposed project aim is to design and development of an app for car driver. The Internet of Things (IOT) is the interconnection of uniquely identifiable embedded computing devices within the existing Internet infrastructure. Typically, IOT offers advanced connectivity of devices, systems, and services that goes beyond machine-to-machine communications (M2M) and covers a variety of protocols[3], domains, and applications. The interconnection of these embedded devices (including smart objects), is implemented in nearly all fields of automation enabling advanced applications like a Smart Grid. The term - things in the IOT refers to a wide variety of devices such as heart monitoring implants, biochip transponders on farm animals, electric clams in coastal waters, automobiles with built-in sensors, or field operation devices that assist fire-fighters in search and rescue[4]. Current market

${ }^{1}$ Department. Of Computer Science \& Engineering SDM Institute of Technology, Ujire, Karnataka, India

${ }^{2}$ Department. Of Computer Science \& Engineering SDM Institute of Technology, Ujire, Karnataka, India

${ }^{3}$ Department. Of Computer Science \& Engineering SDM Institute of Technology, Ujire, Karnataka, India 
examples include thermostat systems and washer/dryers that utilize Wi-Fi for remote monitoring[5].

In this project we are presenting an IoT based system which will help drivers to drive the car safely and efficiently[6]. This system consists of tracking and locating the location of accident using GPS and communicates the co-ordinates via SMS using onboard GSM module, smoke detection using a gas sensor, obstacle detection in front and rear of car using ultrasonic sensor and simulation of physically unavailable sensors using logical functions.

Raspberry pi: Raspberry $\mathrm{Pi}$ is a small, powerful, cheap, hackable and education-oriented computer board introduced in 2012. This credit card-sized computer with many performances and affordable for $25-35 \$$ is perfect platform for interfacing with many devices. The Raspberry Pi board contains a processor and graphics chip, program memory (RAM) and various interfaces and connectors for external devices. Some of these devices are essential, others are optional but all Raspberry Pi models have the same CPU named BCM2835 which is cheap, powerful, and it does not consume a lot of power. Raspberry Pi operates in the same way as a standard PC, requiring a keyboard for command entry, a display unit and a power supply.SD Flash memory card normally used in digital cameras is configured in such a way to 'look like' a hard drive to Raspberry Pi's processor. The unit is powered via the micro USB connector. Internet connectivity may be via an Ethernet/LAN cable or via an USB dongle (Wi-Fi connectivity). The Raspberry $\mathrm{Pi}$ is a small device coming in at $85.60 \mathrm{~mm} \times 53.98 \mathrm{~mm} \times 17 \mathrm{~mm}$ and weighing only 45g. $\square$ The $3.5 \mathrm{~mm}$ analog audio jack allows connecting headphones and speakers to the Raspberry Pi. The High Definition Multi-media Interface (HDMI) port allows the Raspberry Pi to be hooked up to high-definition televisions and monitors that support the technology.

Arduino: Arduino is an open-source electronics platform based on easy-to-use hardware and software. It's intended for anyone making interactive projects. Arduino senses the environment by receiving inputs from many sensors, and affects its surroundings by controlling lights, motors, and other actuators. The Arduino board contains a microcontroller, program memory (SRAM) and various interfaces and connectors for external devices.

\section{LITERATURE SURVEY}

In past, the following works were carried out by some people.

2.1 Gurjashan Singh Pannu, Mohammad Dawud and Pritha Gupta, "Design and Implementation of Autonomous Car using Raspberry Pi”- ( 2015),

In this paper focus is on building a monocular vision autonomous car prototype using Raspberry $\mathrm{Pi}$ as a processing chip. An HD camera along with an ultrasonic sensor is used to provide necessary data from the real world to the car. The car is capable of reaching the given destination safely and intelligently thus avoiding the risk of human errors. Many existing algorithms like lane detection, obstacle detection are combined together to provide the necessary control to the car.

2.2 Sumit Garethiya1, Lohit Ujjainiya and Vaidehi Dudhwadkar, "predictive vehicle collision avoidance system using Raspberry - Pi"- (2015)

In this paper, an effective method is proposed for the collision avoidance system of a vehicle to detect the obstacle present in front and blind spot of the vehicle. The driver is made alert via a buzzer and LED indication as the distance between vehicle and obstacle reduces and is displayed on display board. The ultrasonic sensor detects the state of the object whether it is in motion or 
static with respect to the vehicle. This system is useful for detecting vehicle, motorcycle, bicycle and pedestrians that pass by the lateral side of vehicle.

2.3 V.Sagar Reddy, Dr.L.Padma Sree, and V. Naveen Kumar, "Design and Development of accelerometer based System for driver safety" - (2014)

This paper presents a new design of commodity hardware with cheap and it consumes very less power designed oriented product for getting information from accident location of driver drowsiness and indicating be alert to the driver in the prevention of accident. This system is designed by using Raspberry Pi (ARM11) for fast accessing to control and accelerometer for event detection. Is there any event is occurs the message sent to the authorized person so they can take immediate action to save the lives and reduce the damages.

2.4 M.H Mohamad, Mohd Amin Bin Hasanuddin, Mohd Hafizzie Bin Ramli, "Vehicle Accident Prevention System Embedded with Alcohol Detector" - (2013)

An efficient system of vehicle accident prevention system embedded by alcohol detector has been proposed. This system capable to alert the driver about the level of drunkenness by indicates the condition on LCD display. It also produce an alarm from buzzer to make the driver aware their own condition and to vigilant other people in surrounding area. The most safety element provided by this system is the driver in high level of drunkenness is not allowed to drive a car as the ignition system will be deactivated.

\section{PROPOSED SYSTEM}

In this project it presents an IoT based system which will help the drivers to drive the car safely and efficiently. This system consists of tracking and locating the location of accident using GPS and communicates the co-ordinates via SMS using onboard GSM module, smoke detection using a gas sensor, obstacle detection in front and rear of car using ultrasonic sensor and simulation of physically unavailable sensors using logical functions. The proposed system is designed to be able to housed in a vehicle, main processing unit of the system is Raspberry pi board which handles all data accumulation, analysis of that data and taking actions based the data.

\section{Architecture:}

System consists of array of sensors ultrasonic sensors at the front and the rear, an MQ-3 alcohol sensor for alcohol consumption detection, a smoke sensor to detect fire or engine part malfunction, a triple axes accelerometer is mounted on the system to detect collisions and car positional stability. And system comprises of GSM (Global System for Mobile communication) and GPS (Global Positioning System) for emergency assistance, in the event of any emergency positional data which is obtained from GPS module is communicated to a pre-registered phone number via SMS using GSM module. 


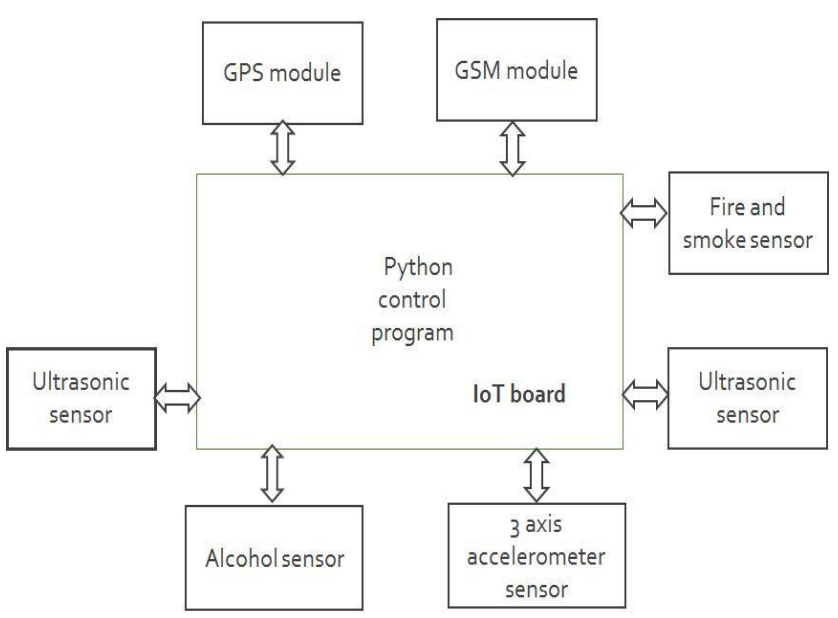

Fig 1: Architecture of working model

All the sensors and modules are connected directly to raspberry pi board to its GPIO (General Purpose Input Output) pins, and a centralized program monitors sensors and issues actions based on their status.

\section{User interface:}

System comprises of a comprehensive and intuitive python programmed GUI, it consists of set of buttons to activate and give input data to logical sensors, when a button is pressed a pop-up window appears with text box for data reception, and after the data is processed status of the particular logical sensor is displayed in an engine status window. And for physical sensors which are interfaced to the raspberry pi there are buttons to activate them, they continuously monitor, and when a notification has to be displayed it is displayed in the engine status part of the main window.

Smoke and alcohol sensor (mq2 and mq3):

Proposed system with the gas sensor It can detect LPG, I-butane, methane, alcohol, Hydrogen, smoke and so on. Based on its fast response time. Measurements can be taken as soon as possible.
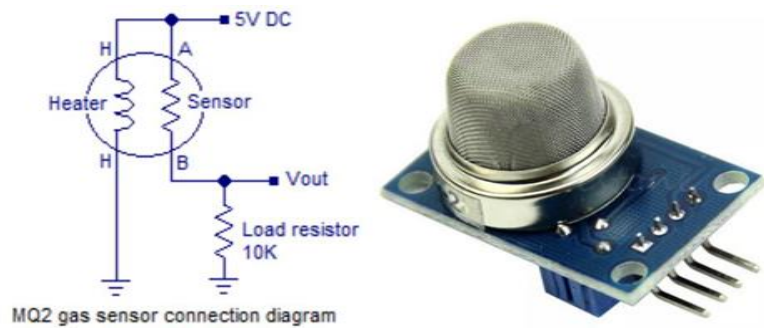

Fig 2

Working: Sensitive material of gas sensor is $\mathrm{SnO} 2$, which with lower conductivity in clean air. When the target combustible gas exist, The sensors conductivity is more higher along with the 
gas concentration rising. Sensor Converts change of conductivity to correspond output signal of gas concentration, gas sensor has high sensitivity to LPG, Propane and Hydrogen, also could be used to Methane and other combustible steam. And MQ-3 is sensitive to ethanol.

GPS MODULE:

GPS stands for Global Positioning System, in the proposed system GPS is used to keep track of the location of the vehicle and send coordinates of the vehicle to a pre-registered phone number using a GSM module.
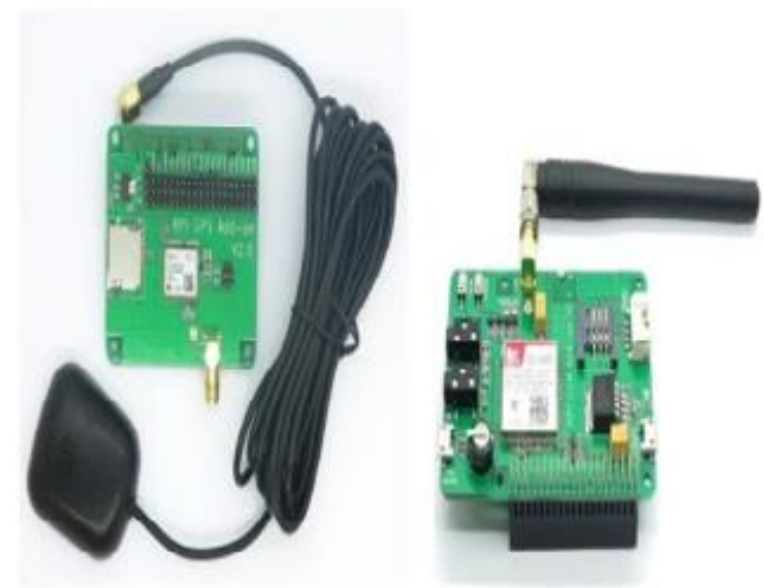

Fig 3: GPS Module

Working: The Global Positioning System consists of a network of 24 broadcasting satellites orbiting the earth at a height of $20,200 \mathrm{~km}$. GPS also consists of receivers on the ground, which listen to and interpret the transmissions of the satellites. Stations on the earth carefully monitor the orbit of each satellite, maintaining a highly accurate record of the satellites instantaneous position. The knowledge of the precise position of the satellites allows them to be used as reference points, from which GPS receivers on earth can determine their position. This technique of determining the position of an object is called ranging.

\section{IV.ALGORITHMS}

The following are some of the algorithms that are implemented in this paper.

\section{Traffic lights:}

Step 1: read light status

Step 2: compare

If light->red

Print "stop engine"

Else if light-> yellow

Print "start engine"

Else

Print "ready to go" 


\section{Pulse monitor:}

Step 1: read heart rate

Step 2: if heart rate < threshold rate and heart rate $>$ threshold rate

Step 3: if heart rate $>$ normal rate and heart rate $<$ normal rate

Advice to take rest.

\section{Temperature Sensor:}

Step 1: read temperature and weather type

Step 2: if weather is rainy

Actuator-> switch on wiper

Else if weather is snowy

Display message to avoid route

Else if weather is foggy

Actuator->switch on fog lamps

Else

Display normal

\section{Algorithms used for Physical sensors}

Some of the physical sensor algorithms are as follows

\section{Obstacle detection:}

To initiate operation we must set pulse length of 10us to the TRIG pin. This causes sensor transmit eight cycles of ultrasonic burst at 40khz and then stopping the pulse to the TRIG pin shuts it off. Then listen to the ECHO pin continuously until ECHO pin becomes TRUE this instant is taken as signal-on; other variable signal-off is taken after transmission from sensor is completed.

\section{Time elapsed=signalon - signaloff}

\section{Algorithm:}

Step 1:initiate sensor by sending a pulse length of 10 micro sec at trig pin.

Step 2: if an obstacle is present waves will bounce back and received at echo pin.

Step 3: calculate time elapsed

Time $=$ signal on-signal off

Step 4: Calculate distance

Distance=elapsed $* 34000$

Distance $=$ distance $/ 2$

Step 5: if distance < threshold value

Alert driver

Smoke and Alcohol Detector (MQ2 and MQ3):

Step 1 : initiate MQ2 sensor

Step 2 : while true

Step 3 : if sensor finds any smoke

Alert driver 


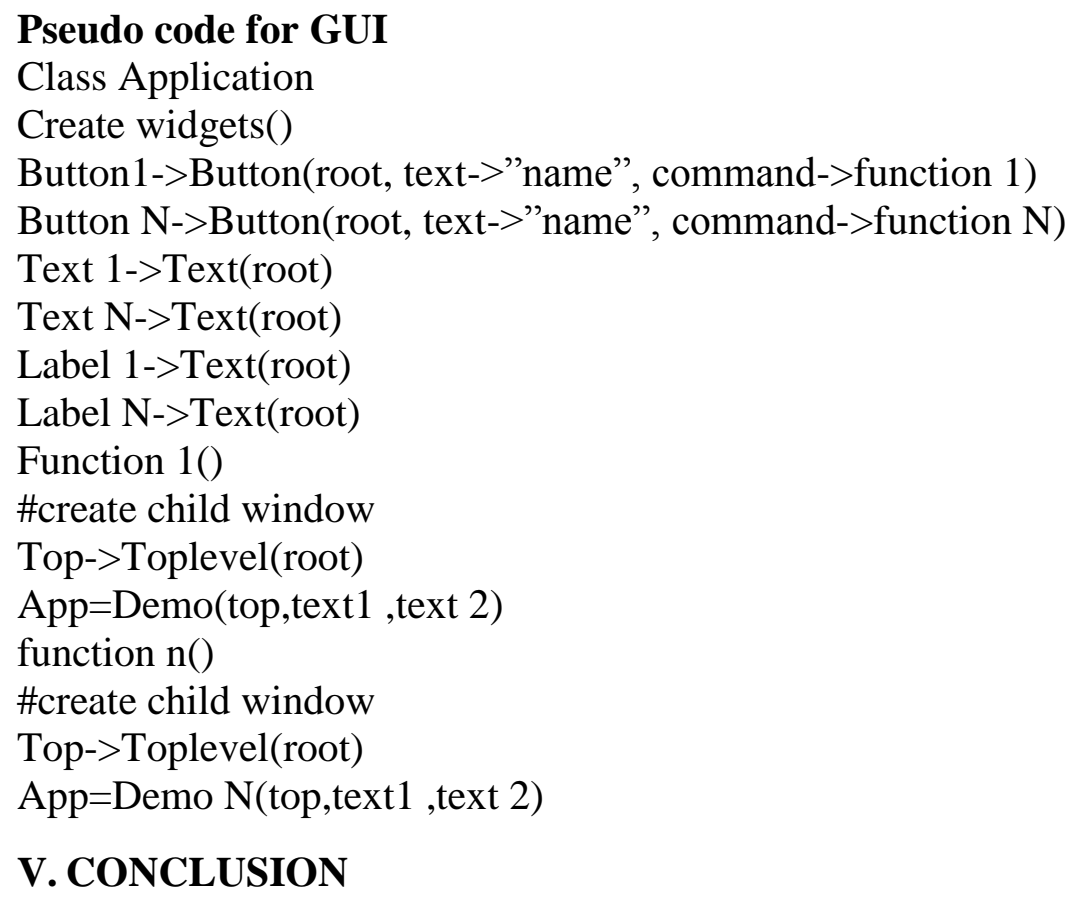

Vehicular Accidents has always been a major catastrophe since the inception of Transport System, Statistics shows huge amount of innocent lives claimed by these accidents and Vehicular accidents have seen surge in recent years, advancement of technology can be used to deploy safety system.

This system is a step in the direction of safe driving of vehicles, it uses cheap and reliable Raspberry pi as central controlling board and is interfaced with sensors for obstacle detection, Alcohol consumption detection, and accelerometer for accident detection and board uses data from these sensors to determine emergency situations and can ask for assistance using GSM module with coordinates from GPS module. And also system also uses many logical sensors due to unavailability or due to cost effectiveness.

\section{REFERENCES}

[1] Gurjashan Singh Pannu, Mohammad Dawud and Pritha Gupta “Design P. S. Huang, C. S. Chiang, C. P. Chang, and T. M. Tu, "Robust spatial watermarking technique for colour images via direct saturation adjustment," Vision, Image and Signal Processing, IEE Proceedings -, vol. 152, pp. 561-574, 2005.

[2] V.Sagar Reddy, Dr.L.Padma Sree , and V. Naveen Kumar, "Design and Development of accelerometer based System for driver safety”, International Journal of Science, Engineering and Technology Research (IJSETR), Volume 3, Issue 12, December 2014

[3] M.H Mohamad, Mohd Amin Bin Hasanuddin, Mohd Hafizzie Bin Ramli, "Vehicle Accident Prevention System Embedded with Alcohol Detector", International Journal of Review in Electronics \& Communication Engineering (IJRECE),Volume 1 - Issue 4 October 2013

[4] A. Bar Hillel, R. Lerner, D. Levi, \& G. Raz."Recent progress in road and lane detection: a survey." Machine Vision and Applications, Feb. 2012, pp. 727-745

[5] Narayan Pandharinath Pawar, and Minakshee M.Patil, “Driver Assistance System based on Raspberry Pi”, International Journal of Computer Applications, Volume 95- No.16, June 2014.

[6] Sumit Garethiya1, Lohit Ujjainiya and Vaidehi Dudhwadkar, "Predictive vehicle collision avoidance system using Raspberry - Pi”, ARPN Journal of Engineering and Applied Sciences, Vol. 10, May 2015. 